\title{
Three dimensional higher spin holography
}

\section{Lovrekovic*}

The Blackett Laboratory, Imperial College London

Prince Consort Road, London SW7 2AZ, United Kingdom E-mail: i lovreko i c . ac . uk

We study the three point coupling for two scalars and particle of arbitrary spin in the $A d S_{3} / C F T_{2}$ holography from the side of holographic reconstruction approach.

*Thanks to Stefan Fredenhagen

Corfu Summer Institute 2017 Training School "Quantum Spacetime and Physics Models" 16 September - 23 September, 2017 


\section{Introduction and motivation for higher spin theory}

Higher spin (HS) theory is an interesting theory that can thank its recent popularity to its possibility of answering important questions in string theory. It was introduced by Vasilliev [1] and one can consider it as a missing link in the evolution from the field theories of lower spins to superstring theories. It is an important symmetry since it does not result from the symmetry breaking. Due to the fact there is no energy scale that describes it, it can be viewed as a toy model for the fundamental, beyond Planck scale theory [2].

It has encountered a number of "no-go" theorems through history because one cannot find a consistent HS theory on the flat space. The solution came through consideration of the AdS space. In the AdS background there is a solution to an HS gravitational interactions up to cubic order which appear in the action, while if one considers equations of motion, the solution can be found up to all orders.

Using HS theory, one can also learn more about the AdS/CFT duality. Klebanov and Polyakov have conjectured that the HS theory on $A d S_{4}$ is dual to a $2+1$ dimensional $\mathrm{O}(\mathrm{N})$ vector model in the large $\mathrm{N}$ limit.Analogously, in one dimension lower, $A d S_{3} / C F T_{2}$ correspondence has been studied as a correspondence between the Vasiliev theory coupled to pair of complex scalar fields, and t'Hooft limit of the $W_{n}$ minimal model CFT. Where the coset representation of the latter is

$$
\frac{S U(N)_{k} \oplus S U(N)_{1}}{S U(N)_{k+1}}
$$

while the 't Hooft limit is

$$
N, k \rightarrow \infty, \quad \lambda=\frac{N}{k+N}
$$

which is fixed. Duality can be further verified by studying the observables such as partition function, its one loop correction [3], correlation function, global symmetries on the boundary and in the bulk [4], and others. In this talk we focus on the three point correlation function.

Scalar - scalar - higher spin field (00s) correlation function has been computed for the $\mathrm{O}(\mathrm{N})$ vector model using the standard field theory methods and compared to the computation using the holographic reconstruction in [5]. The holographic reconstruction method based on computation of the Witten diagrams is general and one can use it to compute the coupling not only for the $\mathrm{O}(\mathrm{N})$ vector model, but for the model for which one knows to determine the boundary currents. This means that one can consider analogous procedure for more general $\lambda$. (00s) field three point function for general $\lambda$ has been considered in [6]. Here, we describe the holographic reconstruction method and how to determine the coupling of the $(00 \mathrm{~s})$ three point function taking into account the result for the (00s) correlation function in the AdS background of Vasiliev's HS theory for any parameter $\lambda$ [6]. The same coupling ought to be obtained from the linearised Vasiliev's equations of motion. In this work, our motivation, beside describing how to obtain $(00 \mathrm{~s})$ coupling, is to consider whether the holographic reconstruction method can be more convenient for computation of coupling for the higher point functions than using linearised Vasiliev's equations.Earlier, the 00s cubic vertices have been analysed in $[7,8,9,10]$. The higher point functions in flat space have been considered in [11]. 


\section{Holographic reconstruction}

Without restriction to particular scaling dimension $\Delta$ the three point function in ambient space formalism can be obtained using standard methods from CFT side, while from the bulk side, one can determine it using Witten diagrams. If one builds a general conformal structure from the three different points, the structure can parametrised using six objects

$$
Y_{i}=\frac{z_{i} \cdot x_{i, i+1}}{x_{i, i+1}^{2}}-\frac{z_{i} \cdot x_{i, i+2}}{x_{i, i+2}^{2}} \quad H_{i}=\frac{1}{x_{i+1, i+2}^{2}}\left(z_{i+1} \cdot z_{i+2}+\frac{2 z_{i+1} \cdot x_{i+1, i+2} z_{i+2} \cdot x_{i+2, i+1}}{x_{i+1, i+2}^{2}}\right),
$$

for $i=1,2,3$. Conformal symmetry, scale transformations and spin structure determine the general three-point functions obtained from the CFT side

$$
\left\langle J_{s_{1}}\left(x_{1} \mid z_{1}\right) J_{s_{2}}\left(x_{2} \mid z_{2}\right) J_{s_{3}}\left(x_{3} \mid z_{3}\right)\right\rangle=\sum_{n_{i}} C_{s_{1}, s_{2}, s_{3}}^{n_{1}, n_{3}, n_{3}} \frac{Y_{1}^{s_{1}-n_{2}-n_{3}} Y_{2}^{s_{2}-n_{3}-n_{1}} Y_{3}^{s_{3}-n_{1}-n_{2}} H_{1}^{n_{1}} H_{2}^{n_{2}} H_{3}^{n_{3}}}{\left(x_{12}^{2}\right)^{\frac{\tau_{1}+\tau_{2}-\tau_{3}}{2}}\left(x_{23}^{2}\right)^{\frac{\tau_{2}+\tau_{3}-\tau_{1}}{2}}\left(x_{31}^{2}\right)^{\frac{\tau_{3}+\tau_{1}-\tau_{2}}{2}}}
$$

for $\tau_{i}=\Delta_{i}-s_{i}$ twists and coefficients $C_{s_{1}, s_{2}, s_{3}}^{n_{1}, n_{3}}$ which are dependent on the theory. This has for the specific case of the $\mathrm{O}(\mathrm{N})$ vector model been determined in [5]. Here, with $J$ we have denoted currents of higher spin $s_{i}$ for $i=1,2,3$ while the coordinates $x_{i}$ are contracted with the auxiliary vectors $z_{i}$ according to ambient space formalism. $x_{i j}$ denotes the difference of coordinates $x_{i j}=$ $x_{i}-x_{j}$. Determining and normalising the currents and choosing the $00 \mathrm{~s}$ case, for $O_{0}$ scalars and $J_{S}$ higer spin current, one obtains $\left\langle O_{0} O_{0} J_{s}\right\rangle$.

To compare this with the bulk side, one needs to consider the on-shell cubic interaction in the ambient framework. The most general cubic vertex in the AdS background in $d+1$ dimensions is [12]

$$
\mathscr{V}_{s_{1}, s_{2}, s_{3}}=\sum_{n_{i}} g_{s_{1}, s_{2}, s_{3}}^{n_{1} n_{3} n_{3}} I_{s_{1} s_{2} s_{3}}^{n_{1} n_{2} n_{3}}\left(\Phi_{i}\right)
$$

for

$$
I_{s_{1} s_{2} s_{3}}^{n_{1} n_{2} n_{3}}\left(\phi_{i}\right)=\left.\mathscr{Y}_{1}^{s_{1}-n_{2}-n_{3}} \mathscr{Y}_{2}^{s_{2}-n_{3}-n_{1}} \mathscr{Y}_{3}^{s_{3}-n_{1}-n_{2}} \mathscr{H}_{1}^{n_{1}} \mathscr{H}_{2}^{n_{2}} \mathscr{H}_{3}^{n_{3}} \Phi_{1}\left(X_{1} U_{1}\right) \Phi_{2}\left(X_{2} U_{2}\right) \Phi_{3}\left(X_{3} U_{3}\right)\right|_{X_{i}=X}
$$

and

$$
\mathscr{Y}_{Y}=\partial_{U_{i}} \cdot \partial_{X_{i+1}}, \quad \mathscr{H}_{i}=\partial_{U_{i+1}} \cdot \partial_{U_{i+2}} .
$$

These objects, are the bulk quantities that correspond to the six conformal boundary structures (2.1).

Expression (2.3) describes the sum of the couplings $g_{s_{1}, s_{2}, s_{3}}^{n_{1} n_{2} n_{3}}$ multiplied with corresponding amplitudes $I_{s_{1} s_{2} s_{3}}^{n_{1} n_{2} n_{3}}$.

\section{Determination of coupling}

The three point function for the arbitrary $\lambda$ computed from the CFT and AdS side [6] is 


$$
\begin{aligned}
& \left\langle O_{ \pm}\left(z_{1}\right) \bar{O}_{ \pm}\left(z_{2}\right) J^{(s)}\left(z_{3}\right)\right\rangle=\left\langle\mathscr{O} \mathscr{O} J^{(s)}\right\rangle_{\text {gauge symmetries }}= \\
& =\frac{(-1)^{s-1} B_{\phi}}{\left.2 \pi^{2}\left|z_{12}\right|^{2(1 \pm \lambda)}\right)} \frac{\Gamma(s)^{2}}{\Gamma(2 s-1)} \frac{\Gamma(s \pm \lambda)}{\Gamma( \pm \lambda)\left(\frac{z_{12}}{z_{113} z_{23}}\right)^{2}} \\
& =\frac{(-1)^{s-1}}{2 \pi} \frac{\Gamma(s)^{2}}{\Gamma(2 s-1)} \frac{\Gamma(s \pm \lambda)}{\Gamma(1 \pm \lambda)}\left(\frac{z_{12}}{z_{13} z_{23}}\right)^{s}\left\langle O_{ \pm}\left(z_{1}\right) \bar{O}_{ \pm}\left(z_{2}\right)\right\rangle .
\end{aligned}
$$

It is comparable with the result from the holographic reconstruction when we take into account the normalisation of the currents

$$
\left\langle J^{(s)}(z) J^{(s)}(0)\right\rangle=\langle J J\rangle_{\text {gauge symmetries }}=\frac{3}{2^{2 s-1} \pi^{5 / 2}} \frac{\sin (\pi \lambda)}{\lambda\left(1-\lambda^{2}\right)} \frac{\Gamma(s) \Gamma(s-\lambda) \Gamma(s+\lambda)}{\Gamma\left(s-\frac{1}{2}\right)} \frac{1}{z^{2 s}} .
$$

To determine the coupling, one has to take the ratio with the three point function obtained by holographic reconstruction. This result is expected to agree with the coupling studied using the linearised Vasiliev's equations of motion in [13]. There, the coupling has been determined from the linearised equations of motion for the scalar field in the higher spin background field.

\section{Outline}

We have described the procedure for obtaining the coupling of the (00s) three point function for the higher spin theory in $A d S_{3} / C F T_{2}$ holography. The talk is based on current work. Analogous computations could be done for the higher point functions from "holographic reconstruction approach" and from "Vasiliev's equations of motion approach". Computations from one approach tend to be more convenient, therefore they are interesting to be determined for the lower correlation functions, so that one could compute them more efficiently for the higher correlation functions in the future.

\section{Acknowledgements}

I would like to thank Stefan Fredenhagen and Arkady Tseytlin for discussions. The work has been made possible thanks to the University of Vienna, Mathematical Physics Department and thanks to the Corfu Summer Institute 2017 Training School "Quantum Spacetime and Physics Models" and by by the FWF project J-4129 within the framework of Schrödinger Programme.

\section{References}

[1] Mikhail A. Vasiliev. Consistent equation for interacting gauge fields of all spins in (3+1)-dimensions. Phys. Lett., B243:378-382, 1990.

[2] V. E. Didenko and E. D. Skvortsov. Elements of Vasiliev theory. 2014.

[3] Matthias R. Gaberdiel, Rajesh Gopakumar, Thomas Hartman, and Suvrat Raju. Partition Functions of Holographic Minimal Models. JHEP, 08:077, 2011. 
[4] Matthias R. Gaberdiel and Thomas Hartman. Symmetries of Holographic Minimal Models. JHEP, 05:031, 2011.

[5] Charlotte Sleight and Massimo Taronna. Higher Spin Interactions from Conformal Field Theory: The Complete Cubic Couplings. Phys. Rev. Lett., 116(18):181602, 2016.

[6] Martin Ammon, Per Kraus, and Eric Perlmutter. Scalar fields and three-point functions in D=3 higher spin gravity. JHEP, 07:113, 2012.

[7] Anastasios C. Petkou. Evaluating the AdS dual of the critical O(N) vector model. JHEP, 03:049, 2003.

[8] Xavier Bekaert, Johanna Erdmenger, Dmitry Ponomarev, and Charlotte Sleight. Quartic AdS Interactions in Higher-Spin Gravity from Conformal Field Theory. JHEP, 11:149, 2015.

[9] E. D. Skvortsov and Massimo Taronna. On Locality, Holography and Unfolding. JHEP, 11:044, 2015.

[10] Massimo Taronna. Pseudo-local Theories: A Functional Class Proposal. In Proceedings, International Workshop on Higher Spin Gauge Theories: Singapore, Singapore, November 4-6, 2015, pages 59-84, 2017.

[11] R. Roiban and A. A. Tseytlin. On four-point interactions in massless higher spin theory in flat space. 2017.

[12] Euihun Joung and Massimo Taronna. Cubic interactions of massless higher spins in (A)dS: metric-like approach. Nucl. Phys., B861:145-174, 2012.

[13] I. Lovrekovic. Three dimensional higher spin holography. In 17th Hellenic School and Workshops on Elementary Particle Physics and Gravity (CORFU2017) Corfu, Greece, September 2-28, 2017, 2018. 